ISSN 0103-8478

\title{
Linhaça marrom e dourada: propriedades químicas e funcionais das sementes e dos óleos prensados a frio
}

\author{
Brown and golden flaxeed: chemical and functional properties of the \\ seeds and the cold-pressed oils
}

\begin{abstract}
Ana Karina Mauro Barroso ${ }^{\mathrm{I}}{ }^{*}$ Alexandre Guedes Torres ${ }^{\mathrm{I}}$ Vanessa Naciuk Castelo-Branco
Andrea Ferreira ${ }^{I}$ Priscilla Vanessa Finotelli ${ }^{I}$ Suely Pereira Freitas ${ }^{\mathrm{I}}$

Maria Helena Miguez da Rocha-Leão ${ }^{\mathrm{I}}$
\end{abstract}

RESUMO

O objetivo do estudo foi comparar a composição química e a capacidade antioxidante das sementes e dos óleos de linhaça marrom e dourada, e avaliar a estabilidade oxidativa dos óleos. Nas sementes, foram determinados: composição centesimal, capacidade antioxidante total e compostos fenólicos totais. Nos óleos, foram determinados: índice de acidez e peróxido, matéria insaponificável, composição em ácidos graxos, tocoferóis, capacidade antioxidante, compostos fenólicos totais e estabilidade oxidativa em quatro condições de estocagem por até um ano. Não houve diferenças significativas entre os tipos de sementes quanto às variáveis investigadas, e ainda entre os óleos quanto ao rendimento da prensagem, composição em ácidos graxos insaturados, índice de acidez, teores de insaponificáveis e compostos fenólicos totais. Apesar do declínio da estabilidade oxidativa de ambos os óleos durante o armazenamento, o óleo de linhaça marrom e os óleos estocados sob refrigeração apresentaram-se mais estáveis. O índice de peróxido foi maior no óleo de linhaça dourada. O teor de tocoferol total e capacidade antioxidante total foram maiores no óleo de linhaça marrom. Contudo, observou-se uma igualdade entre as sementes de ambas as variedades de linhaça e uma superioridade do óleo de linhaça marrom.

Palavras-chave: linhaça, óleo de linhaça, estabilidade oxidativa, capacidade antioxidante, Linun usitatissimum $L$.

\section{ABSTRACT}

The aim of the study was to compare the chemical composition and antioxidant capacity of the brown and golden flaxseed and their cold-pressed oils, and evaluate the oxidative stability of oils. We determined the chemical composition, total antioxidant capacity and total phenolic compounds of the seeds. In the oils we determined acid and peroxide value, unsaponifiable matter, composition of fatty acids and tocopherols, phenolic compounds, antioxidant capacity and oxidative stability in four storage conditions up to one year. There were no significant differences between the flaxseed types in the analyzed properties and between the oils in terms of yield after pressing, composition of unsaturated fatty acids, acid value, unsaponifiables and phenolic compounds. The oxidative stability of both oils decreased with storage time, however the brown flaxseed oil and the oils stored under refrigeration were more stable. The peroxide value was higher in the golden flaxseed oil. The total tocopherol content and antioxidant capacity were higher in brown flaxseed oil. It was observed equality between the brown and golden flaxseed and a superiority of brown flaxseed oil.

Key words: flaxseed, flaxseed oil, oxidative stability, antioxidant capacity, Linun usitatissimum $L$.

\section{INTRODUÇÃO}

A linhaça (Linun usitatissimun L.) é o alimento de origem vegetal mais rico em ácidos graxos $\omega-3$, apresentando também quantidades elevadas de fibras, proteínas e compostos fenólicos (THOMPSON \& CUNNANE, 2003). Existem duas variedades de linhaça para consumo humano, a linhaça marrom e a linhaça dourada. Sua cor é determinada pela quantidade de pigmentos no revestimento externo da semente (COSKUNER \& KARABABA, 2007), sendo que essa quantidade é determinada por fatores genéticos (GROTH et al., 1970) e ambientais (MORRIS, 2007). Existem evidências de que a linhaça marrom e a dourada são semelhantes em sua composição química (MUELLER et al., 2010), portanto, é possível que ambas apresentem bioatividade similar. No entanto, são escassos

Instituto de Química, Centro de Tecnologia, Universidade Federal do Rio de Janeiro (UFRJ), Av. Athos da Silveira Ramos, 149, Centro de Tecnologia, Cidade Universitária, Ilha do Fundão, 21941-909, Rio de Janeiro, RJ, Brasil. E-mail: kakamauro@yahoo.com.br. *Autor para correspondência. 
estudos que as comparem, especialmente quanto à sua capacidade antioxidante total. Dessa forma, comparar as variedades de linhaça pode contribuir para agregar valor à linhaça marrom, produzida em maior quantidade no país (CAMPOS, 2007) e com menor valor comercial. O presente trabalho teve como objetivo comparar a composição química e a capacidade antioxidante total das sementes e dos óleos de linhaça marrom e linhaça dourada, assim como avaliar a estabilidade oxidativa dos óleos extraídos das sementes.

\section{MATERIAL E MÉTODOS}

Sementes de linhaça marrom e dourada cultivadas no Brasil foram adquiridas no comércio varejista da cidade do Rio de Janeiro-RJ. As sementes foram trituradas em processador doméstico de alimentos (Britânia) e as farinhas armazenadas a $-20^{\circ} \mathrm{C}$ até as análises. Foram determinados teores de umidade, cinzas, lipídeos e proteínas (fator de conversão - 5,7) segundo a AOAC (AOAC, 2005) e teor de fibra detergente neutro (FDN) segundo Van Soest (VAN SOEST, 1963), o qual quantifica os componentes estruturais da parede celular (celulose, hemicelulose e lignina). Os carboidratos totais foram calculados por diferença (AOAC, 2005) e o valor energético total foi calculado através da energia procedente dos nutrientes, considerando os fatores de conversão de Atwater (USP, 2007). Para a determinação dos fenólicos totais e da capacidade antioxidante total das sementes, foi utilizado o extrato hidrofílico obtido após extração das farinhas com metanol na proporção de 3:1 (p/v), seguido de homogeneização, filtração em gaze de algodão, concentração em evaporador rotativo $\left(55^{\circ} \mathrm{C}\right)$ e liofilização. Os compostos fenólicos totais foram determinados utilizando o reagente de FolinCiocalteau (MORAIS et al., 2008) com leitura em 760nm (espectrofotômetro UV-Vis Hitachi U-300). As curvas de calibração foram preparadas com ácido gálico. Os resultados foram expressos como mg de equivalentes de ácido gálico (EAG) por $100 \mathrm{~g}$ de semente (mg EAG $\left.100 \mathrm{~g}^{-1}\right)$. A capacidade antioxidante total foi determinada através do ensaio de atividade sequestrante de DPPH' (radical 2,2-difenil-1-picrilhidrazila) (BRAND-WILLIAMS et al., 1995), com leitura em 518nm (espectrofotômetro Shimadzu UV1600) após uma hora de reação e calculada conforme a Equação (1):

$$
\frac{100-[(\Delta \mathrm{Abs} \times 100)]}{\mathrm{Abs} \text { cont }}
$$

em que $\Delta \mathrm{Abs}$ é a diferença entre a absorbância da amostra e a do branco; $\mathrm{Abs}_{\text {cont }}$ é a absorbância do branco. A capacidade antioxidante total foi calculada por regressão linear para cada extrato, obtendo-se a concentração de extrato necessária para reduzir em $50 \%\left(\mathrm{CE}_{50}\right)$ a concentração inicial do radical DPPH. Todas as análises foram feitas em triplicata.

Os óleos foram extraídos das sementes por prensagem mecânica a frio, em prensa do tipo parafuso sem fim (OEKOTEC, tipo CA59G). Determinou-se o índice de acidez, índice de peróxido, matéria insaponificável (IAL, 2005) e conteúdo de ácidos graxos (AOAC, 2005) sob a forma de ésteres metílicos (HARTMAN \& LAGO, 1973) em cromatógrafo gasoso de alta resolução (VARIAN HP 3800). Para a análise de tocoferóis, as amostras de óleo foram dissolvidas em $n$-hexano, filtradas $(0,45 \mu \mathrm{m})$ (GIMENO et al., 2000) e analisadas por cromatografia líquida de alta eficiência de fase normal (TAN \& BRZUSKIEWICZ, 1989), em cromatógrafo Pharmacia LKB. As quatro formas de tocoferóis ( $\alpha$-, $\beta-, \gamma-$ e $\delta$ ) foram identificadas e quantificadas por meio de padronização externa através de curvas de calibração com padrão comercial de cada tocoferol $\left(\mathrm{r}^{2}>0,98\right)$. Os resultados foram expressos em $\mathrm{mg}$ $100 \mathrm{~g}^{-1}$ de óleo. A capacidade antioxidante total dos óleos foi determinada na amostra integral e nas frações lipofílica e hidrofílica pelo ensaio de TEAC (Trolox Equivalent Antioxidant Capacity), no qual se monitora a capacidade dos compostos antioxidantes presentes na amostra em reduzir o radical pré-formado ABTS' (ácido 2'-azinobis-3etilbenzeno-tiazolina-6-sulfônico). Os resultados foram comparados com aqueles obtidos no ensaio com Trolox. A cinética de redução foi monitorada pela diminuição da absorção do ABTS• em 734nm (espectrofotômetro Shimadzu UV-1800). As frações lipofílica e hidrofílica foram obtidas por extração em hexano e metanol, respectivamente (CASTELOBRANCO \& TORRES, 2012). Os resultados foram expressos como mmoles de equivalente de trolox $\mathrm{kg}^{-1}$ de amostra (mmol TEAC $\mathrm{kg}^{-1}$ ). Os compostos fenólicos totais nos óleos foram extraídos com metanol aquoso $80 \% \quad(\mathrm{v} / \mathrm{v})$ e determinados espectrofotometricamente (espectrofotômetro Shimadzu UV-1800) em 725nm após reação com o reagente de Folin-Ciocalteau (SZYDLOWSKACZERNIAK et al., 2008). Os resultados foram expressos como mg de equivalentes de ácido gálico (EAG) por $\mathrm{kg}$ de óleo (mg EAG kg-1). Para avaliar a estabilidade oxidativa dos óleos, utilizou-se o equipamento Rancimat ${ }^{\circledR} 743$ (Metrohm), conforme método Cd 12b-92 da AOCS (AOCS, 1993), a $110^{\circ} \mathrm{C}$, 
sob fluxo de ar de $10 \mathrm{~L} \mathrm{~h}^{-1}$, utilizando-se $3 \mathrm{~g}$ de óleo. As amostras foram armazenadas por até 12 meses, fracionadas em alíquotas após 1, 2, 4, 6 e 12 meses de armazenamento, nas seguintes condições: vidro claro e temperatura ambiente; vidro claro e refrigeração $\left(8-10^{\circ} \mathrm{C}\right)$; vidro âmbar e temperatura ambiente; vidro âmbar e refrigeração $\left(8-10^{\circ} \mathrm{C}\right)$. A passagem de ar pela amostra induziu a formação de compostos voláteis, coletados em água destilada, os quais alteraram condutividade do meio. $\mathrm{O}$ aumento da condutividade da água em função do acúmulo dos produtos voláteis e do tempo de oxidação permitiu o cálculo de período de indução (PI), expresso em horas. Todas as análises foram feitas em triplicata.

Análise descritiva foi aplicada a todos os dados e os resultados foram apresentados como média (comparadas por teste- $T$ ) e desvio padrão. No ensaio de DPPH', análise de covariância (ANCOVA) foi usada para comparação das curvas de atividade antioxidante. No ensaio de TEAC, a área abaixo da curva (AUC) foi calculada para as curvas de Abs vs. tempo. Essas análises foram executadas no programa GraphPad Prism v. 4. Os fatores determinantes da estabilidade oxidativa dos óleos foram investigados através de modelo linear generalizado no programa Statgraphics v. 12.2. Como variáveis independentes do modelo, utilizou-se o tipo de linhaça, o tipo de embalagem, a temperatura e o período de armazenamento dos óleos; e, como variável dependente, utilizou-se o período de indução (PI). Resultados com valores de $\mathrm{P}<0,05$ foram considerados significativos.

\section{RESULTADOS E DISCUSSÃO}

Não foram observadas diferenças significativas entre as linhaças quanto à composição centesimal (Tabela 1), a qual foi semelhante àquela publicada anteriormente (MORRIS, 2007), exceto quanto ao teor de lipídios, que foi inferior ao de linhaças cultivadas na América do Norte (FLAX COUNCIL OF CANADA, 2001). Em relação à capacidade antioxidante, as sementes não diferiram estatisticamente (Figura 1) e não houve diferença significativa entre as equações ajustadas por regressão linear (ANCOVA, $\mathrm{P}>0,05$ ), sendo uma única equação apresentada no gráfico. Pesquisas sobre capacidade antioxidante da linhaça são escassas, e as existentes apresentam metodologias distintas ou contemplam atividade antioxidante de componentes específicos da semente, como, por exemplo, as lignanas (HOSSEINIAN et al., 2006; GALVÃO et al., 2008). Quanto aos compostos fenólicos totais, não houve diferenças significativas $(\mathrm{P}>0,05)$ entre as sementes de linhaça marrom $(1332 \pm 0,09 \mathrm{mg}$ de EAG $\left.100 \mathrm{~g}^{-1}\right)$ e dourada $\left(1039 \pm 0,21 \mathrm{mg}\right.$ de EAG $\left.100 \mathrm{~g}^{-1}\right)$. VELIOGLU et al. (1998) e BOZAN \& TEMELLI (2008) analisaram fenóis totais em sementes de linhaça e reportaram, respectivamente, 509 e 1670mg de EAG $100 \mathrm{~g}^{-1}$ de semente. Possivelmente, esses resultados divergentes relacionam-se ao estágio de desenvolvimento da semente e/ou a diferenças no clima e solo, relacionadas à sua origem.

O rendimento da extração do óleo de linhaça marrom (36,5\%) e do óleo de linhaça dourada $(35,4 \%)$ não apresentou diferença significativa $(\mathrm{P}>0,05)$. Não houve diferença significativa quanto à qualidade inicial dos óleos, exceto para o índice de peróxido, maior na linhaça dourada (Tabela 2). A acidez de ambos os óleos (Tabela 2) atendeu aos critérios de qualidade da AOCS, que estabelece como satisfatório índice de acidez $<3 \%$ para o óleo bruto de linhaça (FIRESTONE, 2006). O teor médio de $0,77 \%$ de matéria insaponificável para ambas as amostras (Tabela 2) também atendeu aos limites definidos pela

Tabela 1 - Composição centesimal das sementes de linhaça marrom e dourada.

\begin{tabular}{lcc}
\hline & & \\
Fatores (unidade) & Marrom & Dourada \\
\hline Umidade $\left({\left.\mathrm{g} 100 \mathrm{~g}^{-1}\right)}\right.$ & $7,77 \pm 0,37$ \\
Cinzas $\left(\mathrm{g} 100 \mathrm{~g}^{-1}\right)$ & $2,89 \pm 0,14$ & $3,01 \pm 0,11$ \\
Lipídeos $\left(\mathrm{g} 100 \mathrm{~g}^{-1}\right)$ & $33,7 \pm 0,56$ & $34,8 \pm 0,07$ \\
Proteínas $\left(\mathrm{g} 100 \mathrm{~g}^{-1}\right)$ & $19,1 \pm 1,15$ & $21,6 \pm 0,12$ \\
Fibra (FDN; $\left.\mathrm{g} 100 \mathrm{~g}^{-1}\right)$ & $28,0 \pm 4,24$ & $22,5 \pm 4,95$ \\
Carboidrato $\left(\mathrm{g} 100 \mathrm{~g}^{-1}\right)$ & $9,22 \pm 4,75$ & $10,4 \pm 4,55$ \\
Valor energético total $\left(\mathrm{Kcal} 100 \mathrm{~g}^{-1}\right)$ & $417 \pm 6,22$ & $441 \pm 7,25$ \\
\hline
\end{tabular}

FDN: fibra detergente neutro.

Letras sobrescritas diferentes na mesma linha indicam médias diferentes significativamente $(\mathrm{P}<0,05$, teste- $T)$. 


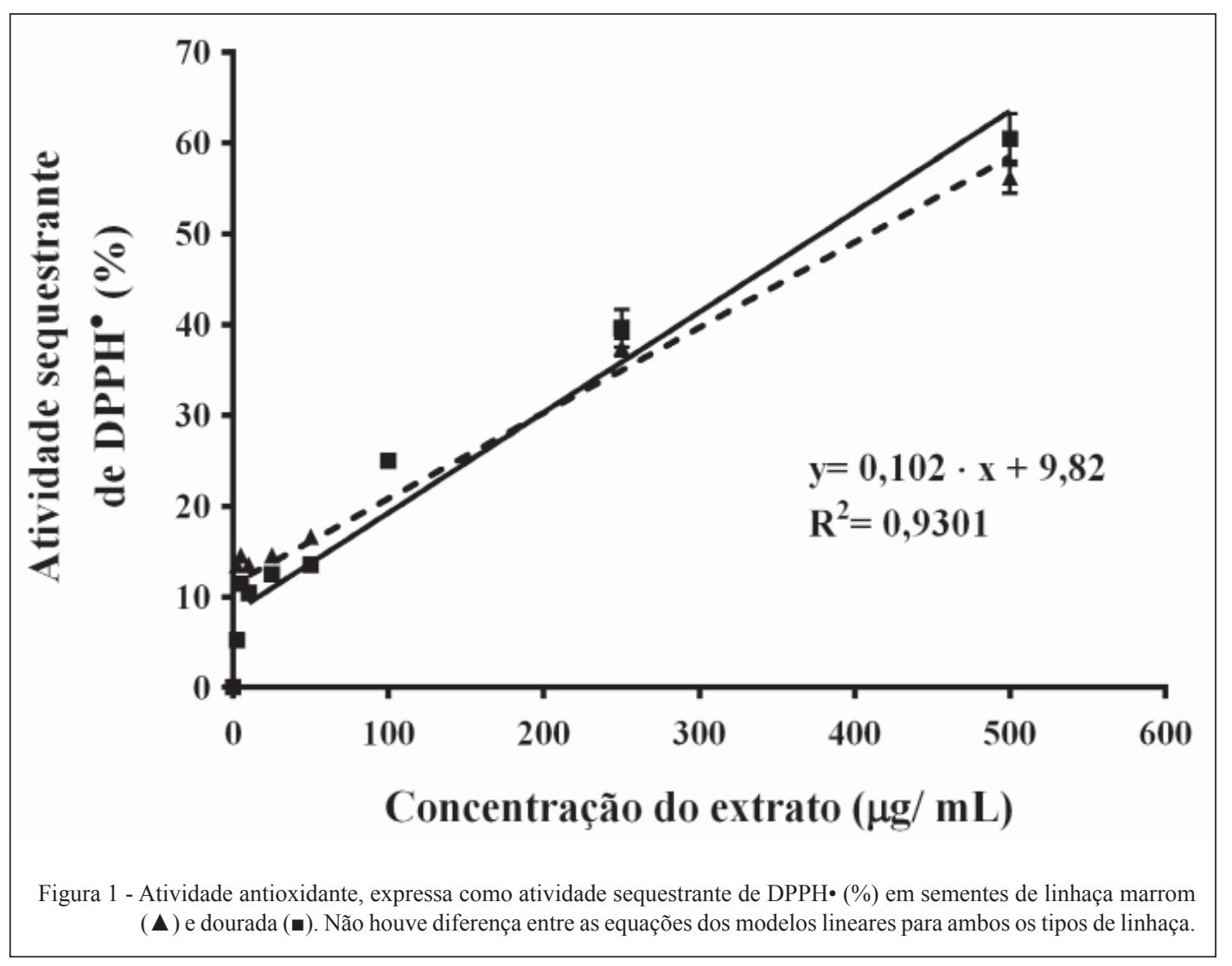

AOCS, que determina matéria insaponificável entre 0,1 e 2\% para o óleo de linhaça (FIRESTONE, 2006). A composição em ácidos graxos foi consistente com dados da AOCS (FIRESTONE, 2006). Houve diferença significativa somente em relação ao conteúdo de ácido esteárico (C18:0), maior na linhaça marrom (Tabela 2). Embora saturado, esse ácido graxo não é aterogênico, por ser rapidamente

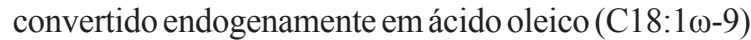
(BONANOME \& GRUNDY, 1988). O óleo de linhaça marrom apresentou maiores teores de tocoferóis totais e de $\gamma$-tocoferol (Tabela 2). Os teores de $\alpha$-tocoferol nas amostras foram aparentemente superiores aos encontrados por TUBEROSO et al. (2007), diferenças que podem estar relacionadas a fatores genéticos e/ou ambientais. Os teores de compostos fenólicos totais dos óleos não indicaram diferença significativa entre si (Tabela 2), entretanto apresentaram-se inferiores $(\mathrm{P}<0,05)$ aos teores observados nas suas respectivas sementes. Possivelmente, houve retenção de compostos fenólicos no resíduo sólido da prensagem (torta), tais como ligninas e lignanas, encontradas na parte fibrosa da semente (THOMPSON \& CUNNANE, 2003). Além disso, os teores de compostos fenólicos totais observados no presente estudo foram inferiores aos reportados anteriormente (HERCHI et al., 2011), possivelmente devido à menor eficiência da prensagem a frio na extração desses componentes em comparação à extração com solventes, usada por HERCHI et al. (2011). Uma maior capacidade antioxidante total foi observada no óleo de linhaça marrom (Tabela 2) devido, possivelmente, aos seus maiores teores de tocoferóis. Examinando os valores de TEAC nas amostras integrais e frações, verificaram-se valores superiores na fração lipofílica de ambos os óleos (Tabela 2), demonstrando, provavelmente, que a capacidade antioxidante destes deva-se, sobretudo, aos seus teores de tocoferóis presentes na fração lipofílica, em detrimento dos compostos fenólicos totais presentes na fração hidrofílica. A capacidade antioxidante de ambas as amostras foram superiores aos resultados reportados anteriormente (TUBEROSO et al., 2007), diferenças que podem ser atribuídas aos protocolos experimentais, como, por exemplo, ao radical e/ou aos solventes empregados no ensaio. A estabilidade dos óleos, representada pelo período de indução (PI) em horas, reduziu com o armazenamento em todas as condições de estocagem (Figura 2) e foi dependente da temperatura de armazenamento e do tipo de 
Tabela 2 - Qualidade inicial, composição em ácidos graxos, tocoferóis, compostos fenólicos totais e capacidade antioxidante total em óleos de linhaça marrom e dourada.

\begin{tabular}{|c|c|c|}
\hline \multirow[t]{2}{*}{ Fatores (unidade) } & \multicolumn{2}{|c|}{ Óleos de linhaça (média \pm DP)- } \\
\hline & Marrom & Dourada \\
\hline Matéria insaponificável $\left({\left.\mathrm{g} 100 \mathrm{~g}^{-1}\right)}^{-1}\right.$ & $0,77 \pm 0,12$ & $0,77 \pm 0,31$ \\
\hline Índice de acidez (g ác. Oleico $100 \mathrm{~g}^{-1}$ ) & $1,41 \pm 0,11$ & $1,51 \pm 0,09$ \\
\hline Índice de peróxido $\left(\right.$ meq $\left.\mathrm{O}_{2} \mathrm{~kg}^{-1}\right)$ & $0,50 \pm 0,04^{\mathrm{a}}$ & $1,22 \pm 0,13^{\mathrm{b}}$ \\
\hline \multicolumn{3}{|l|}{ Ácidos Graxos Saturados $\left({\left.\mathrm{g} 100 \mathrm{~g}^{-1}\right)}\right.$} \\
\hline Ácido mirístico (C14:0) & $0,03 \pm 0,08$ & $0,03 \pm 0,08$ \\
\hline Ácido palmítico (C16:0) & $4,90 \pm 0,12$ & $4,28 \pm 0,14$ \\
\hline Ácido esteárico (C18:0) & $2,61 \pm 0,09^{b}$ & $0,91 \pm 0,14^{\text {a }}$ \\
\hline Total & $7,60 \pm 0,11^{b}$ & $5,24 \pm 0,18^{a}$ \\
\hline \multicolumn{3}{|l|}{ Ácidos Graxos Insaturados $\left({\left.\mathrm{g} 100 \mathrm{~g}^{-1}\right)}\right.$} \\
\hline Ácido miristoleico (C14:1) & $0,01 \pm 0,02$ & $0,01 \pm 0,09$ \\
\hline Ácido palmitoleico (C16:1) & n.d. & $0,02 \pm 0,01$ \\
\hline Ácido oleico $(\mathrm{C} 18: 1 \omega-9)$ & $20,4 \pm 0,22$ & $23,0 \pm 0,30$ \\
\hline 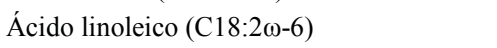 & $13,3 \pm 0,17$ & $13,4 \pm 0,26$ \\
\hline 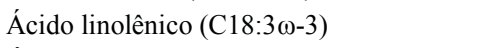 & $58,4 \pm 0,14$ & $58,3 \pm 0,10$ \\
\hline Ácido erúcico (C22:1) & $0,09 \pm 0,01$ & n.d. \\
\hline Total & $92,4 \pm 0,23$ & $94,8 \pm 0,14$ \\
\hline Razão $\omega-6 / \omega-3$ & 0,23 & 0,23 \\
\hline \multicolumn{3}{|l|}{ Tocoferóis $\left(\mathrm{mg} 100 \mathrm{~g}^{-1}\right)$} \\
\hline$\alpha$-tocoferol & $7,58 \pm 0,90$ & $7,41 \pm 0,91$ \\
\hline$\beta$-tocoferol & $13,8 \pm 0,31$ & $15,0 \pm 1,74$ \\
\hline$\gamma$-tocoferol & $38,9 \pm 0,64^{b}$ & $25,5 \pm 4,21^{\mathrm{a}}$ \\
\hline Total & $60,3 \pm 0,44^{\mathrm{b}}$ & $47,9 \pm 1,82^{\mathrm{a}}$ \\
\hline Compostos fenólicos totais $\left(\mathrm{mg} \mathrm{EAG} \mathrm{kg}{ }^{-1}\right.$ ) & $4,3 \pm 0,06$ & $4,1 \pm 0,05$ \\
\hline \multicolumn{3}{|c|}{ Capacidade antioxidante total $\left(\mathrm{mmol}\right.$ TEAC $\left.\mathrm{kg}^{-1}\right)$} \\
\hline Fração Hidrofílica & $3,76 \pm 0,21$ & $3,41 \pm 0,24$ \\
\hline Fração Lipofílica & $5,15 \pm 0,77^{\mathrm{b}}$ & $3,54 \pm 0,43^{\text {a }}$ \\
\hline Amostra Integral & $6,15 \pm 0,32^{b}$ & $4,41 \pm 0,35^{\text {a }}$ \\
\hline
\end{tabular}

EAG: equivalentes de ácido gálico. TEAC: Trolox Equivalent Antioxidant Capacity.

Letras sobrescritas diferentes na mesma linha indicam médias diferentes significativamente $(\mathrm{P}<0,05$, teste- $T)$. n.d.: não-detectado.

linhaça, de acordo com o modelo linear generalizado ajustado (Equação 2; $\mathrm{R}^{2}$ ajustado=0,8513; $\mathrm{P}=0,0000$ ).

$$
\mathrm{PI}=3,475-0,1038 \times C_{\text {Tipo }}+0,2465 \times C_{\text {Temp. }}-0,006595 \times \text { tempo }
$$

em que, PI é o período de indução (horas); $\mathrm{C}_{\text {Tipo }}=$ 1 , se linhaça dourada ou -1 , se linhaça marrom; $\mathrm{C}_{\text {Temp }}=1$, se armazenado a $8^{\circ} \mathrm{C}$ ou -1 se armazenado em temperatura ambiente; tempo é o período de armazenamento (dias). Os resultados do teste de Durbin-Watson (DW) indicaram bom ajuste do modelo linear generalizado. Conforme esperado, o tempo de estocagem influenciou negativamente a estabilidade oxidativa e a refrigeração aumentou a estabilidade dos óleos. Quanto ao tipo de linhaça, observou-se que a linhaça marrom foi a mais estável no armazenamento, resultado consistente com seu maior conteúdo de tocoferóis e capacidade antioxidante.

\section{CONCLUSÃO}

As sementes de linhaça marrom e dourada e seus respectivos óleos foram similares quanto à maior parte dos fatores investigados. No entanto, as diferenças observadas nos óleos foram favoráveis ao de linhaça marrom, que apresentou maior conteúdo de C18:0, maior teor de tocoferóis, maior capacidade antioxidante e maior estabilidade oxidativa. Dessa forma, a linhaça marrom, particularmente seu óleo, apresentou qualidade superior à linhaça dourada. Portanto, o consumo da linhaça marrom, produzida 


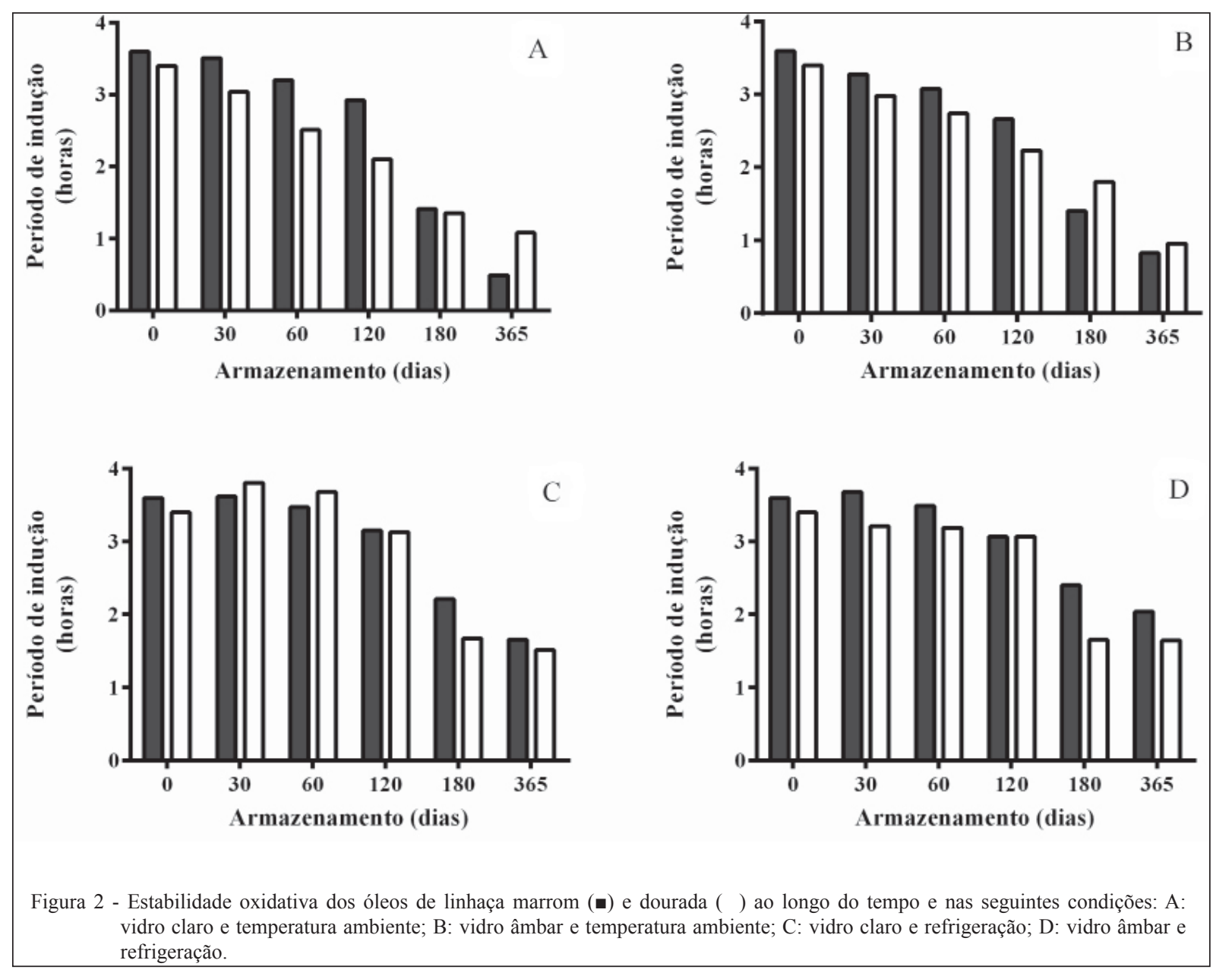

em maior quantidade no Brasil e com menor valor comercial, deve ser incentivado com o intuito de aumentar a funcionalidade da dieta brasileira.

\section{REFERÊNCIAS}

AOCS (AMERICAN OIL CHEMISTS SOCIETY). Official Methods and Recommended Practices of the AOCS. 4.ed. Champaign, Illinois: AOCS, 1993. 1200p.

AOAC (ASSOCIATION OF OFFICIAL ANALYTICAL CHEMISTS INTERNATIONAL). Official Methods of Analysis of the Association Analytical Chemists. 18.ed. Gaitherburg, 2005. $1526 \mathrm{p}$.

BONANOME, A.; GRUNDY, S.M. Effect of dietary stearic acid on plasma cholesterol and lipoprotein levels. New England Journal of Medicine, v.318, n.19, p.1244-1248, 1988. Disponível em: <http://www.nejm.org/doi/full/10.1056/ NEJM198805123181905>. Acesso em: 13 ago. 2011. doi. 10.1056/NEJM198805123181905.

BOZAN, B.; TEMELLI, F. Chemical composition and oxidative stability of flax, safflower and poppy seed and seed oils. Bioresource of Technology, v.99, n.14, 6354-6359, 2008. Disponível em: $<\mathrm{http}: / /$ www.ncbi.nlm.nih.gov/pubmed/18198133>. Acesso em: 12 maio 2011. doi. 10.1016/j.biortech.2007.12.009 pp.6354-9.

BRAND-WILLIAMS, W. et al. Use of a free radical method to evaluate antioxidant activity. Lebensmittel- Wissenschaft und Technologie, v.28, n.1, p.25-30, 1995. Disponível em: <http:// www.ncbi.nlm.nih.gov/pubmed/18198133>. Acesso em: 05 maio 2010. doi. 10.1016/j.biortech.2007.12.009.

CAMPOS, V.M.C. Produção e beneficiamento de sementes de linhaça. Fundação Centro Tecnológico de Minas Gerais CETEC, 2007. Disponível em: <http://www.sbrt.ibict.br>. Acesso em: 20 abr. 2010.

CASTELO-BRANCO, V.N.; TORRES, A.G. Generalized linear model describes determinants of total antioxidant capacity of refined vegetable oils. European Journal of Lipid Science and Technology, v.114, n.3, p.332-342, 2012. Disponível em: <http:// onlinelibrary.wiley.com/doi/10.1002/ejlt.201100181/abstract>. Acesso em: 11 nov. 2012. doi. 10.1002/ejlt.201100181.

COSKUNER, Y.; KARABABA, E. Some physical properties of flaxseed (Linum. usitatissimum L.). Journal of Food Engineering, v.78, n.3, p.1067-1073, 2007. Disponível em: $<$ http:// www.sciencedirect.com/science/article/pii/S0260877406000136>. 
Acesso em: 10 abr. 2010. doi. 10.1016/j.jfoodeng.2005.12.017. FIRESTONE, D. Physical and chemical characteristics of oils, fats and waxes. 2.ed. Washington, D.C: AOCS, 2006. 237p.

FLAX COUNCIL OF CANADA. Brown flax seeds, or golden? 2001. Disponível em: $<$ http://www.flaxcouncil.ca/english/index. jsp?p=g7\&mp=nutrition $>$. Acesso em: 20 abr. 2009.

GALVÃO, E.L. et al. Avaliação do potencial antioxidante e extração subcrítica do óleo de linhaça. Ciência e Tecnologia Alimentos, v.28, n.3, p.551-557, 2008. doi. 10.1590/S010120612008000300008 .

GIMENO, E. et al. Rapid determination of vitamin E in vegetable oils by reversed-phase high-performance liquid chromatography. Journal of Chromatography A, v.881, n.1-2, p.251-254, 2000. Disponível em: <http://www.sciencedirect.com/science/article/pii/ S0021967300002193>. Acesso em: 18 mar. 2011. doi. 10.1016/ S0021-9673(00)00219-3.

GROTH, J.V. et al. Effect of seed color on tolerance of flax to seedling blight caused by Rhizoclonia solani. Phytopathology, v.60, p.379-380, 1970.

HARTMAN, L.; LAGO, R.C.A. Rapid preparation of fatty acid methyl ester from lipids. Laboratory Practice, v.22, n.6, p.475476, 1973.

HERCHI, W. et al. Changes in the content of phenolic compounds in flaxseed oil during development. Journal of the American Oil Chemists' Society, v.88, n.8, p.1135-1142, 2011. Disponível em: $<$ http://link.springer.com/article/10.1007\%2Fs11746-011-1783-2>. Acesso em: 19 nov. 2011. doi. 10.1007/s11746-011-1738-2.

HOSSEINIAN, F.S. et al. Antioxidant capacity of flaxseed lignans in two model systems. Journal of the American Oil Chemists' Society, v.83, n.10, p.835- 840, 2006. doi. 10.1007/s11746-006-5034-x.

IAL (INSTITUTO ADOLFO LUTZ). Normas analíticas do Instituto Adolfo Lutz: métodos químicos e físicos para análise de alimentos. 4.ed. Brasília: ANVISA, 2005. 533p.

MORAIS, S.A.L. et al. Análise de compostos bioativos, grupos ácidos e da atividade antioxidante do café arábica (Coffea arabica) do cerrado e de seus grãos defeituosos (PVA) submetidos a diferentes torras. Ciência e Tecnologia de Alimentos, v.28, Suppl, p.198-207, 2008. Disponível em: <http://www.scielo.br/pdf/cta/ v28s0/31.pdf>. Acesso em: 10 fev. 2010. doi. 10.1590/S010120612008000500031.
MORRIS, D.H. Flax: a health and nutrition primer. 4.ed. Winnipeg: Flax Council of Canada, 2007. 140p.

MUELLER, K. et al. Functional properties and chemical composition of fractionated brown and yellow linseed meal (Linum usitatissimum L.). Journal of Food Engineering, v.98, n.4, p.453-460, 2010. Disponível em: <http://www.sciencedirect. com/science/article/pii/S0260877410000440>. Acesso em: 10 ago. 2010. doi. 10.1016/j.jfoodeng.2010.01.028.

SZYDLOWSKA-CZERNIAK,A. et al. Determination of antioxidant capacities of vegetable oils by ferric-ion spectrophotometric methods. Talanta, v.76, n.4, p.899-905, 2008. Disponível em: $<$ http://www.ncbi.nlm.nih.gov/pubmed/18656676>. Acesso em: 25 jun. 2011. doi. 10.1016/j.talanta.2008.04.055.

TAN, B.; BRZUSKIEWICZ, L. Separation of tocopherol and tocotrienol isomers using normal- and reverse-phase liquid chromatography. Analytical Biochemistry, v.180, n.2, p.368-373, 1989. Disponível em: <http://www.sciencedirect.com/science/ article/pii/0003269789904478>. Acesso em: 12 ago. 2011. doi. 10.1016/0003-2697(89)90447-8.

THOMPSON, L.U.; CUNNANE, S.C. Flaxseed in human nutrition. 2.ed. Champaign, Illinois: AOCS, 2003. 458p.

TUBEROSO, C.I.G. et al. Determination of antioxidant compounds and antioxidant activity in commercial oilseeds for food use. Food Chemistry, v.103, n.4, p.1494-1501, 2007. Disponível em: <http://www.sciencedirect.com/science/article/pii/ S0308814606006716>. Acesso em: 04 jul. 2011. doi. 10.1016/j. foodchem.2006.08.014

UNIVERSIDADE DE SÃO PAULO (USP). Tabela brasileira de composição dos alimentos. Campinas, 2007. Disponível em: <http:www.fcf.usp.br/>. Acesso em: 12 mar. 2009.

VAN SOEST, P.J. Use of detergents in the analysis of fibrous foods. II. A rapid method for the determination of fibre and lignin. Journal of the Association of the Official Analytical Chemists, v.46, p.829-835, 1963. Disponível em:<http:/garfield.library. upenn.edu/ classics1979/A1979HZ28600001.pdf>. Acesso em: 04 fev. 2010.

VELIOGLU, Y.S. et al. Antioxidant activity and total phenolics in selected fruits, vegetables, and grain products. Journal of Agricultural and Food Chemistry, v.46, n.10, p.4113-4117, 1998. Disponível em: <http://pubs.acs.org/doi/abs/10.1021/ jf9801973>. Acesso em: 08 set. 2011. doi. 10.1021/jf9801973. 